\title{
Global bifurcation for asymptotically linear Schrödinger equations
}

\author{
François Genoud
}

\begin{abstract}
We prove global asymptotic bifurcation for a very general class of asymptotically linear Schrödinger equations

$$
\left\{\begin{array}{l}
\Delta u+f(x, u) u=\lambda u \quad \text { in } \mathbb{R}^{N}, \\
u \in H^{1}\left(\mathbb{R}^{N}\right) \backslash\{0\}, \quad N \geqslant 1 .
\end{array}\right.
$$

The method is topological, based on recent developments of degree theory. We use the inversion $u \rightarrow v:=u /\|u\|_{X}^{2}$ in an appropriate Sobolev space $X=W^{2, p}\left(\mathbb{R}^{N}\right)$, and we first obtain bifurcation from the line of trivial solutions for an auxiliary problem in the variables $(\lambda, v) \in \mathbb{R} \times X$. This problem has a lack of compactness and of regularity, requiring a truncation procedure. Going back to the original problem, we obtain global branches of positive/negative solutions 'bifurcating from infinity'. We believe that, for the values of $\lambda$ covered by our bifurcation approach, the existence result we obtain for positive solutions of (1) is the most general so far.
\end{abstract}

Mathematics subject classification (2010). Primary 35J61; Secondary 35B32.

Keywords. Asymptotically linear Schrödinger equations, Semilinear elliptic eigenvalue problems, Global bifurcation, Unbounded domains.

\section{Introduction}

We consider nonlinear Schrödinger equations of the form

$$
\left\{\begin{array}{l}
\Delta u+f(x, u) u=\lambda u \quad \text { in } \mathbb{R}^{N}, \\
u \in H^{1}\left(\mathbb{R}^{N}\right) \backslash\{0\}, \quad N \geqslant 1,
\end{array}\right.
$$

where the nonlinearity is supposed to be asymptotically linear in the sense that there exists $f_{\infty} \in C\left(\mathbb{R}^{N}\right)$ such that $f(x, s) \rightarrow f_{\infty}(x)$ as $|s| \rightarrow \infty$ for all

This work was supported by the Engineering and Physical Sciences Research Council, [EP/H030514/1]. 
$x \in \mathbb{R}^{N}$. A solution to (1) is a couple $(\lambda, u) \in \mathbb{R} \times H^{1}\left(\mathbb{R}^{N}\right) \backslash\{0\}$ satisfying the elliptic equation in the sense of distributions. We will establish global bifurcation for (1) in $\mathbb{R} \times W^{2, p}\left(\mathbb{R}^{N}\right)$ with $p \in[2, \infty) \cap\left(\frac{1}{2} N, \infty\right)$, yielding solutions with $u \in H^{1}\left(\mathbb{R}^{N}\right)$. More precisely, we will show that there exist unbounded connected sets $\mathcal{S}^{ \pm} \subset \mathbb{R} \times W^{2, p}\left(\mathbb{R}^{N}\right)$ of positive/negative solutions of (1). We use a method introduced in [17], based on a topological degree for compact perturbations of $C^{1}$ Fredholm maps. We have applied this method recently to problem (1) in dimension $N=1$-actually, on the half-line - [3], by establishing global bifurcation in $H^{2}(0, \infty)$. The present work extends the results of [3] in various directions, as explained in more detail below.

To present the method and discuss our results, let us first rewrite (1) as

$$
\Delta u+f_{\infty}(x) u+g(x, u) u=\lambda u \quad \text { with } \quad g(x, s):=f(x, s)-f_{\infty}(x) .
$$

Following Rabinowitz [11] and Toland [18], we use the inversion $u \rightarrow v:=$ $u /\|u\|^{2}$ (with $\left.\|\cdot\|:=\|\cdot\|_{W^{2, p}\left(\mathbb{R}^{N}\right)}\right)$ to get

$$
\Delta v+f_{\infty}(x) v+g\left(x, v /\|v\|^{2}\right) v=\lambda v,
$$

for which we shall prove global bifurcation from the point $\left(\lambda_{\infty}, 0\right)$ in $\mathbb{R} \times$ $W^{2, p}\left(\mathbb{R}^{N}\right)$. Here, $\lambda_{\infty}>0$ is characterized as the principal eigenvalue of the linear problem

$$
\Delta u+f_{\infty}(x) u=\lambda u,
$$

known as the 'asymptotic linearization' of (1). Returning to the original variable will yield an unbounded connected set of solutions $(\lambda, u)$ of $(1)$, satisfying

$$
\|u\| \rightarrow \infty \quad \text { as } \quad \lambda \rightarrow \lambda_{\infty} .
$$

This behaviour is often referred to as asymptotic bifurcation and was established in $[11,18]$ in the context of boundary value problems in bounded domains. The core of the method there is Rabinowitz's global bifurcation theory. The present situation is more difficult because the domain is unbounded and problem (1) is not asymptotically linear in the rigorous sense - which amounts to saying that the auxiliary problem (3) is not Fréchet differentiable at $v=0$. Following Stuart and Zhou [17], we therefore resort to a truncation procedure to transform (3) into a problem for which global bifurcation can be obtained by applying an abstract theorem proved in [17], based on recent developments of degree theory [12].

Our precise hypotheses on the nonlinearity are the following.

(f1) $f \in C\left(\mathbb{R}^{N} \times \mathbb{R}\right) \cap L^{\infty}\left(\mathbb{R}^{N} \times \mathbb{R}\right)$ and $f(x, \cdot) \in C^{1}(\mathbb{R})$ for all $x \in \mathbb{R}^{N}$.

(f2) We have $f(x, s) \rightarrow f_{0}(x):=f(x, 0)$ as $s \rightarrow 0$, uniformly for $x \in \mathbb{R}^{N}$.

(f3) There exists a function $f_{\infty} \in C\left(\mathbb{R}^{N}\right)$ such that $f(x, s) \rightarrow f_{\infty}(x)$ as $|s| \rightarrow$ $\infty$, uniformly for $x \in \mathbb{R}^{N}$. Furthermore, there exists $\delta>0$ such that

$$
0<f_{0}(x)+\delta<\lambda_{*}:=\limsup _{|x| \rightarrow \infty} f_{\infty}(x)<\infty \quad \text { for all } x \in \mathbb{R}^{N} .
$$

(f4) $0 \leqslant f(x, s) \leqslant f_{\infty}(x)$ for all $(x, s) \in \mathbb{R}^{N} \times \mathbb{R}$.

(f5) $\partial_{2} f(\cdot, s) \in L^{\infty}\left(\mathbb{R}^{N}\right)$ for all $s \in \mathbb{R}$ and $\left\{\partial_{2} f(x, \cdot)\right\}_{x \in \mathbb{R}^{N}}$ is equicontinuous. 
(f6) We have

$$
\lambda_{\infty}:=-\inf _{u \in H^{1}\left(\mathbb{R}^{N}\right) \backslash\{0\}} \frac{\int_{\mathbb{R}^{N}}\left\{|\nabla u|^{2}-f_{\infty}(x) u^{2}\right\} \mathrm{d} x}{\int_{\mathbb{R}^{N}} u^{2} \mathrm{~d} x}>\lambda_{*} .
$$

Remark 1. (i) Note that the hypothesis (f3) prevents $f$ from being independent of $s$.

(ii) Assumption (f6) is satisfied provided $f_{\infty} \underset{\geqslant}{\geqslant} \lambda_{*}$ on $\mathbb{R}^{N}$, in which case the 'limsup' in (f3) is actually a ' $\lim ^{\prime}$ ' $\lim _{|x| \rightarrow \infty} f_{\infty}(x)=\lambda_{*}$.

Theorem 1. Let $f$ satisfy the hypotheses (f1)-(f6) and $p \in[2, \infty) \cap\left(\frac{1}{2} N, \infty\right)$. There exist two connected sets $\mathcal{S}^{ \pm} \subset \mathbb{R} \times W^{2, p}\left(\mathbb{R}^{N}\right)$ of positive/negative solutions of (1) with the following properties.

(i) $\mathrm{P} \mathrm{S}^{ \pm}=\left(\lambda_{*}, \lambda_{\infty}\right)$, where $\mathrm{P}(\lambda, u):=\lambda$ for all $(\lambda, u) \in \mathbb{R} \times W^{2, p}\left(\mathbb{R}^{N}\right)$.

(ii) $\mathcal{S}^{ \pm}$are bounded away from $\mathbb{R} \times\{0\}$ in $\mathbb{R} \times W^{2, p}\left(\mathbb{R}^{N}\right)$.

(iii) If $\left\{\left(\lambda_{n}, u_{n}\right)\right\} \subset \mathcal{S}^{ \pm}$is such that $\lambda_{n} \rightarrow \lambda$ as $n \rightarrow \infty$, then

$$
\lim _{n \rightarrow \infty}\left\|u_{n}\right\|_{L^{p}\left(\mathbb{R}^{N}\right)}=\lim _{n \rightarrow \infty}\left\|u_{n}\right\|_{L^{\infty}\left(\mathbb{R}^{N}\right)}=\infty \Longleftrightarrow \lambda=\lambda_{\infty}
$$

(iv) For $(\lambda, u) \in \mathcal{S}^{ \pm}$, we have $u \in C^{1}\left(\mathbb{R}^{N}\right)$ and $|\nabla u(x)|, u(x) \rightarrow 0$ as $|x| \rightarrow \infty$.

Example 1. The function

$$
f(x, s):=\frac{1}{1+s} f_{0}(x)+\frac{s}{1+s} f_{\infty}(x)
$$

satisfies (f1)-(f6) if $f_{0}, f_{\infty} \in C\left(\mathbb{R}^{N}\right) \cap L^{\infty}\left(\mathbb{R}^{N}\right)$ with $f_{0}, f_{\infty} \geqslant 0, f_{0} \leqslant f_{\infty}$, $f_{\infty} \underset{\equiv}{\geqslant} \lambda_{*}:=\lim _{|x| \rightarrow \infty} f_{\infty}(x)$ and $f_{0}+\delta<\lambda_{*}$ on $\mathbb{R}^{N}$, for some $\delta>0$.

In our previous work on the one-dimensional case [3], we had in mind applications to nonlinear waveguides and were therefore working under more restrictive assumptions. In particular, we were dealing with a nonlinearity of the form $f\left(x, u^{2}\right) u$ with an $f \in C\left(\mathbb{R}_{+}^{2}\right)$ satisfying similar assumptions to (f1)-(f6), but with $f_{0} \equiv$ const. As will be seen in the proof of Theorem 1 , the nonlinearity $f(x, u) u$ in (1) can be handled similarly, without any symmetry assumption. The restriction on $f_{0}$ was made in connection with our previous work on related problems [2], with a view to continuing the branch of solutions down to the line of trivial solutions, where we expect it to meet the $\lambda$-axis at the boundary of the essential spectrum of the linearization at $u=0$. The linearization at $u=0$ and the asymptotic linearization (4) can respectively be written as

$$
L_{0 / \infty} u=\lambda u,
$$

where $L_{0 / \infty}: D\left(L_{0 / \infty}\right):=H^{2}\left(\mathbb{R}^{N}\right) \subset L^{2}\left(\mathbb{R}^{N}\right) \rightarrow L^{2}\left(\mathbb{R}^{N}\right)$ are the Schrödinger operators $^{1}$ defined by

\footnotetext{
${ }^{1}$ As shown in Proposition 3 below, we do not lose any generality by restricting the domain of these operators to $H^{2}\left(\mathbb{R}^{N}\right)$.
} 


$$
L_{0 / \infty} u:=\Delta u+f_{0 / \infty}(x) u .
$$

We refer the reader to [13] for the spectral theory of Schrödinger operators we shall need here. In particular, under the above hypotheses, we have

$$
\lambda_{\infty}=\sup \sigma\left(L_{\infty}\right), \sigma_{\mathrm{e}}\left(L_{\infty}\right) \subset\left(-\infty, \lambda_{*}\right] \text { and } \sigma\left(L_{0}\right) \subset\left(-\infty, \lambda_{*}\right),
$$

where $\sigma$ and $\sigma_{\mathrm{e}}$ denote the spectrum and the essential spectrum, respectively. (Note that $\sigma_{\mathrm{e}}\left(L_{\infty}\right)=\left(-\infty, \lambda_{*}\right]$ if $f_{\infty}$ satisfies the assumption in Remark 1 (ii).) In the present work, as in [3], we make the hypothesis (f6), ensuring that $\lambda_{\infty}$ is an eigenvalue of (4), to obtain a branch of solutions 'bifurcating from infinity'. The discussion regarding bifurcation from the line of trivial solutions relies on $L_{0}$, and two different scenarios can occur, depending on whether $\sup \sigma_{\mathrm{e}}\left(L_{0}\right)<\sup \sigma\left(L_{0}\right)$ or $\sup \sigma_{\mathrm{e}}\left(L_{0}\right)=\sup \sigma\left(L_{0}\right)$. In the first case, $\sup \sigma\left(L_{0}\right)$ is a simple eigenvalue, and the problem can be dealt with by standard bifurcation theory. The one-dimensional problem was studied in great detail in [6]. In the situation where $\sup \sigma_{\mathrm{e}}\left(L_{0}\right)=\sup \sigma\left(L_{0}\right)$ (which occurs for instance if $f_{0} \equiv$ const.), we expect to observe bifurcation from the essential spectrum of $L_{0}$. This case deserves further attention and [3] can be seen as a first step in this direction. However, the method used in [3] is very powerful and allows one to deal with the present, much more general situation.

Asymptotically linear Schrödinger equations have been extensively studied for the past 15 years, see e.g. [1,3,7-10,14-17,19-22]. Most of these papers make use of variational methods to prove existence of solutions of various asymptotically linear problems, many of which can be put in the form (1). Important developments were achieved in order to apply the mountain pass theorem without the so-called 'superquadraticity condition', which is not satisfied by asymptotically linear nonlinearities. Two landmark contributions in this direction were $[7,15]$, but many others followed. In this context, fairly strong assumptions are usually made, requiring for instance radial symmetry, i.e. $f(x, s)=f(|x|, s)$, or simply that $f$ does not depend on $x$, or that at least one of the linear problems (5) is autonomous, i.e. $f_{0}$ or $f_{\infty}$ is constant. To ensure the compactness of minimizing sequences, the concentrationcompactness principle is often used, requiring the existence of a limit problem as $|x| \rightarrow \infty$. At least, limits of $f_{0 / \infty}$ as $|x| \rightarrow \infty$ are usually supposed to exist. In this respect, our work is probably most closely related to [22], where both linearizations are non-autonomous (see also [16] for a one-dimensional problem). Typical results provide existence of positive solutions obtained as critical points of an appropriate functional, for any given $\lambda \in\left(\sup \sigma\left(L_{0}\right), \sup \sigma\left(L_{\infty}\right)\right)$. As far as we know, the only bifurcation-type results were obtained in [17] for a problem with a potential well, where the parameter $\lambda$ appears with a weight. Our method, derived from [17], does not allow $\lambda$ to go below $\lambda_{*}$ since the Fredholm property needs to be preserved along the branch (see Lemma 5 ), and so we do not cover the full interval $\left(\sup \sigma\left(L_{0}\right), \sup \sigma\left(L_{\infty}\right)\right)$. However, for $\lambda \in\left(\lambda_{*}, \lambda_{\infty}\right)$, we believe that our results are the most general so far. In particular, we do not need any symmetry or monotonicity assumptions, and $f_{0 / \infty}$ need not have limits as $|x| \rightarrow \infty$. 
Notation and terminology. Throughout the paper, the usual norm on $L^{r}\left(\mathbb{R}^{N}\right)$ for $1 \leqslant r \leqslant \infty$ will be denoted by $|\cdot|_{r}$. Completely continuous nonlinear mappings between Banach spaces will be termed 'compact'.

\section{Preliminary results}

We first establish some basic properties of solutions of (1).

Proposition 2. Suppose that (f1)-(f6) are satisfied.

(i) If $(\lambda, u) \in \mathbb{R} \times H^{1}\left(\mathbb{R}^{N}\right)$ satisfies (1) then $u \in W^{2, p}\left(\mathbb{R}^{N}\right)$ for all $p \in[2, \infty)$. In particular, $u \in C^{1}\left(\mathbb{R}^{N}\right)$ with $|\nabla u(x)|, u(x) \rightarrow 0$ as $|x| \rightarrow \infty$.

(ii) If $(\lambda, u) \in \mathbb{R} \times W^{2, p}\left(\mathbb{R}^{N}\right)$ satisfies (1) with $\lambda>\lambda_{*}$ and $p \in[2, \infty) \cap$ $\left(\frac{1}{2} N, \infty\right)$, then $u \in H^{1}\left(\mathbb{R}^{N}\right)$.

Proof. If $(\lambda, u)$ is as in (i), $u \in W^{2, p}\left(\mathbb{R}^{N}\right)$ for all $p \in[2, \infty)$ follows by standard elliptic regularity theory (see e.g. [13]), using the fact that $f$ is bounded.

If $(\lambda, u)$ is as in (ii), it follows by Lemma 7 below that $u \in L^{2}\left(\mathbb{R}^{N}\right)$ and so $u \in H^{2}\left(\mathbb{R}^{N}\right) \subset H^{1}\left(\mathbb{R}^{N}\right)$ by elliptic regularity.

We now give some properties of the linear eigenvalue problem

$$
\left\{\begin{array}{l}
\Delta u+f_{\infty}(x) u=\lambda u \quad \text { in } \mathbb{R}^{N}, \\
u \in H^{1}\left(\mathbb{R}^{N}\right) \backslash\{0\}, \quad \lambda>0 .
\end{array}\right.
$$

A number $\lambda>0$ is called an eigenvalue if there exists $u \in H^{1}\left(\mathbb{R}^{N}\right) \backslash\{0\}$, called an eigenfunction, such that $(\lambda, u)$ satisfies (6) (in the sense of distributions).

Proposition 3. Suppose that (f3) and (f6) are satisfied.

(i) If $(\lambda, u) \in \mathbb{R} \times H^{1}\left(\mathbb{R}^{N}\right)$ satisfies (6) then $u \in W^{2, p}\left(\mathbb{R}^{N}\right)$ for all $p \in[2, \infty)$. In particular, $u \in C^{1}\left(\mathbb{R}^{N}\right)$ with $|\nabla u(x)|, u(x) \rightarrow 0$ as $|x| \rightarrow \infty$.

(ii) The number $\lambda_{\infty}$ defined in (f6) is a simple eigenvalue of (6) and there exists a corresponding eigenfunction $\varphi_{\infty} \in H^{1}\left(\mathbb{R}^{N}\right)$ with $\left|\varphi_{\infty}\right|_{2}=1$ and $\varphi_{\infty}>0$ on $\mathbb{R}^{N}$.

Proof. (i) follows by elliptic regularity. As discussed in the introduction, (ii) follows from the spectral theory of Schrödinger operators (see e.g. [13]).

\section{Truncation and bifurcation}

For $p \in[2, \infty) \cap\left(\frac{1}{2} N, \infty\right)$ fixed, we define the following Banach spaces:

$$
\begin{array}{lll}
X:=W^{2, p}\left(\mathbb{R}^{N}\right) & \text { with } & \|\cdot\|_{X}:=\|\cdot\|_{W^{2, p}}, \\
Y:=L^{p}\left(\mathbb{R}^{N}\right) & \text { with } & \|\cdot\|_{Y}:=|\cdot|_{p} .
\end{array}
$$

We start by giving an operator formulation of the inverted problem (3). For $\lambda \in \mathbb{R}$, we define $L(\lambda), G: X \rightarrow Y$ by

$$
L(\lambda) v:=\Delta v+f_{\infty}(x) v-\lambda v, \quad G(v):=g\left(x, v /\|v\|_{X}^{2}\right) v \quad \text { for } v \neq 0, \quad G(0):=0 .
$$


Here, as usual, $u \rightarrow g(x, u)$ denotes the Nemytskii operator generated by $g$. Under the hypotheses of Theorem 1, $G$ is well-defined and continuous. Then (3) is equivalent to

$$
L(\lambda) v+G(v)=0 .
$$

The following global bifurcation theorem is due to Stuart and Zhou. For arbitrary Banach spaces $X$ and $Y$, we denote by $B(X, Y)$ the space of bounded linear operators from $X$ to $Y$, and we let

$$
\Phi_{0}(X, Y):=\{L \in B(X, Y): L \text { is a Fredholm operator of index zero }\} .
$$

Theorem 4. [17, Theorem A.1] Let $L \in C^{1}\left(J, \Phi_{0}(X, Y)\right)$ where $J \subset \mathbb{R}$ is an open interval and $\lambda_{0} \in J$ be such that

$$
\operatorname{dim} \operatorname{ker} L\left(\lambda_{0}\right) \text { is odd and }\left[L^{\prime}\left(\lambda_{0}\right) \operatorname{ker} L\left(\lambda_{0}\right)\right] \oplus \operatorname{rge} L\left(\lambda_{0}\right)=Y \text {. }
$$

Suppose that $K \in C(X, Y)$ is compact and Fréchet differentiable at $u=0$ with $K^{\prime}(0)=0$. Let

$$
\mathcal{Z}:=\{(\lambda, u) \in J \times X: u \neq 0 \text { and } L(\lambda) u+K(u)=0\},
$$

endowed with the metric inherited from $\mathbb{R} \times X$, and let $\mathcal{C}$ denote the connected component of $\mathcal{Z} \cup\left\{\left(\lambda_{0}, 0\right)\right\}$ such that $\left(\lambda_{0}, 0\right) \in \mathcal{C}$. Then $\mathcal{C}$ has at least one of the following properties:

(a) $\mathcal{C}$ is an unbounded subset of $\mathbb{R} \times X$;

(b) $\overline{\mathcal{C}} \cap[J \times\{0\}] \neq\left\{\left(\lambda_{0}, 0\right)\right\}$, where $\overline{\mathcal{C}}$ denotes the closure of $\mathcal{C}$ in $\mathbb{R} \times X$;

(c) either $\inf \mathrm{P} \mathcal{C}=\inf J$ or $\sup \mathrm{P} \mathcal{C}=\sup J$.

In the present context, we would like to obtain informations about connected sets of solutions of problem (7) by applying Theorem 4 with $\lambda_{0}=\lambda_{\infty}, K=G, X, Y$ and $L(\lambda)$ as defined above. Unfortunately, this cannot be done directly because $G$ is not differentiable at $v=0$ (this can be proved similarly to [17, Lemma B.1]) and is not compact. Therefore, we introduce the following sequence of approximate problems. We define $G_{n}: X \rightarrow Y$ by

$$
G_{n}(v)(x):=\chi_{n}(x) G(v)(x) \text { for } v \in X \text { and } n \in \mathbb{N}:=\{1,2, \ldots\},
$$

where

$$
\chi_{n}(x):= \begin{cases}1 & \text { if }|x| \leqslant n, \\ 0 & \text { if }|x|>n .\end{cases}
$$

Since the mapping $\lambda \rightarrow L(\lambda)$ is of class $C^{1}(\mathbb{R}, B(X, Y))$, the following lemma shows that $L(\lambda)$ satisfies the hypotheses of Theorem 4 , with $J=\left(\lambda_{*}, \infty\right)$ and $\lambda_{0}=\lambda_{\infty}$.

Lemma 5. Suppose that (f3) and (f6) are satisfied and let $J=\left(\lambda_{*}, \infty\right)$.

(i) $L(\lambda) \in \Phi_{0}(X, Y)$ for all $\lambda \in J$.

(ii) For $\lambda=\lambda_{\infty} \in J$, we have

$\operatorname{dim} \operatorname{ker} L\left(\lambda_{\infty}\right)=1 \quad$ and $\quad\left[L^{\prime}\left(\lambda_{\infty}\right) \operatorname{ker} L\left(\lambda_{\infty}\right)\right] \oplus \operatorname{rge} L\left(\lambda_{\infty}\right)=Y$. 
Proof. These are standard properties of the Schrödinger operators $L(\lambda)$. Part (i) follows for instance from [5, Sect. 4]. For any $\lambda \in \mathbb{R}, L^{\prime}(\lambda) v=-v$ for all $v \in X$ and $L(\lambda) \in B(X, Y)$ is self-adjoint. Hence (ii) follows from Proposition $3(\mathrm{ii})$.

Our next lemma establishes the hypotheses of Theorem 4 for the truncated operators $G_{n}$.

Lemma 6. Suppose that $f$ satisfies (f1)-(f5), and let $g$ be defined by (2). Then, for all $n \in \mathbb{N}$, the operator $G_{n}$ defined in (8) has the following properties.

(i) $G_{n} \in C(X, Y) \cap C^{1}(X \backslash\{0\}, Y)$.

(ii) $G_{n}$ is Fréchet differentiable at $u=0$ with $G_{n}^{\prime}(0)=0$.

(iii) $G_{n}$ is compact.

Proof. Using the fact that $X \subset L^{r}\left(\mathbb{R}^{N}\right)$ for all $2 \leqslant r \leqslant \infty$, the proof is very similar to that of [3, Lemmas 4 and 5] and so we omit it here. section.

We will suppose that the hypotheses (f1)-(f6) hold for the rest of this

For $n \in \mathbb{N}$ and $J:=\left(\lambda_{*}, \infty\right)$, define $F_{n}: J \times X \rightarrow Y$ by

$$
F_{n}(\lambda, v):=L(\lambda) v+G_{n}(v)
$$

and let

$$
\begin{aligned}
& \mathcal{Z}_{n}:=\left\{(\lambda, v) \in J \times X: v \neq 0 \text { and } F_{n}(\lambda, v)=0\right\}, \\
& \mathcal{C}_{n}:=\text { connected component of } \mathcal{Z}_{n} \cup\left\{\left(\lambda_{\infty}, 0\right)\right\} \text { containing the point }\left(\lambda_{\infty}, 0\right) .
\end{aligned}
$$

We already know from Lemma 5 and Lemma 6 that we can apply Theorem 4 to $F_{n}$ for any $n \in \mathbb{N}$, with $\lambda_{0}=\lambda_{\infty}$. Before doing so, we will first establish some preliminary properties of the sets $\mathcal{Z}_{n}$. We start with exponential decay estimates.

Lemma 7. Let $(\lambda, v) \in J \times X$ satisfy $v L(\lambda) v \geqslant 0$ on $\mathbb{R}^{N}$. For any $\epsilon \in\left(0, \lambda-\lambda_{*}\right)$, there exists $C_{\epsilon}>0$ such that

$$
|v(x)| \leqslant|v|_{\infty} \exp \left[-\left(\lambda-\lambda_{*}-\epsilon\right)^{1 / 2}\left(|x|-C_{\epsilon}\right)\right] \quad \text { for all } x \in \mathbb{R}^{N} .
$$

Proof. The proof is similar to that of [17, Lemma 3.4] but we give it here for completeness. By the definition of $\lambda_{*}$ in (f3), for any $\epsilon \in\left(0, \lambda-\lambda_{*}\right)$, there exists $C_{\epsilon}>0$ such that

$$
|x| \geqslant C_{\epsilon} \Longrightarrow f_{\infty}(x) \leqslant \lambda_{*}+\epsilon<\lambda .
$$

Now let $\eta:=\lambda-\lambda_{*}-\epsilon>0$ and define

$$
z(x):=|v|_{\infty} \mathrm{e}^{-\sqrt{\eta}\left(|x|-C_{\epsilon}\right)}-v(x), \quad \Omega_{\epsilon}:=\left\{x \in \mathbb{R}^{N}:|x| \geqslant C_{\epsilon}, z(x)<0\right\} .
$$

For $x \in \Omega_{\epsilon}$, we have $v(x) \geqslant 0$ and so by our hypothesis and by (9),

$$
\begin{aligned}
0 & \leqslant L(\lambda) v(x)=\Delta v(x)-\lambda v(x)+f_{\infty}(x) v(x) \\
& \Longrightarrow \Delta v(x) \geqslant\left[\lambda-f_{\infty}(x)\right] v(x) \geqslant \eta v(x) \text { for all } x \in \Omega_{\epsilon} .
\end{aligned}
$$


Hence,

$$
\begin{aligned}
\Delta z(x) & =|v|_{\infty} \mathrm{e}^{-\sqrt{\eta}\left(|x|-C_{\epsilon}\right)}\left(\eta-\sqrt{\eta} \frac{N-1}{|x|}\right)-\Delta v(x) \\
& \leqslant|v|_{\infty} \mathrm{e}^{-\sqrt{\eta}\left(|x|-C_{\epsilon}\right)}\left(\eta-\sqrt{\eta} \frac{N-1}{|x|}\right)-\eta v(x) \\
& \leqslant \eta z(x)<0 \quad \text { for all } x \in \Omega_{\epsilon} .
\end{aligned}
$$

Furthermore, $z(x)=|v|_{\infty}-v(x) \geqslant 0$ for $|x|=C_{\epsilon}$ and $z(x) \rightarrow 0$ as $|x| \rightarrow \infty$. Assuming that $\Omega_{\epsilon} \neq \varnothing$, it follows by the weak maximum principle (see e.g. [4, Theorem 8.1]) that $z \geqslant 0$ on $\Omega_{\epsilon}$, a contradiction. Therefore, $\Omega_{\epsilon}=\varnothing$ and

$$
v(x) \leqslant|v|_{\infty} \mathrm{e}^{-\sqrt{\eta}\left(|x|-C_{\epsilon}\right)} \text { for }|x| \geqslant C_{\epsilon} .
$$

Applying a similar argument with $-v$ instead of $v$ shows that

$$
-v(x) \leqslant|v|_{\infty} \mathrm{e}^{-\sqrt{\eta}\left(|x|-C_{\epsilon}\right)} \quad \text { for }|x| \geqslant C_{\epsilon}
$$

as well, and so $|v(x)| \leqslant|v|_{\infty} \mathrm{e}^{-\sqrt{\eta}\left(|x|-C_{\epsilon}\right)}$ for $|x| \geqslant C_{\epsilon}$. Since the inequality is obvious for $|x| \leqslant C_{\epsilon}$, this completes the proof.

The following a priori bounds play a central role in the limit procedure.

Lemma 8. There is a constant $A>0$ such that, for any $\mu \in J$, there exists $N_{\mu} \in \mathbb{N}$ such that

$$
\forall \lambda \geqslant \mu \forall n \geqslant N_{\mu} \quad(\lambda, v) \in \mathcal{Z}_{n} \Longrightarrow\|v\|_{X} \leqslant A .
$$

Proof. For $\mu \in J=\left(\lambda_{*}, \infty\right)$, it follows from (f3) that there exists $N_{\mu} \in \mathbb{N}$ such that

$$
|x|>N_{\mu} \quad \Longrightarrow \quad 0 \leqslant f_{\infty}(x)<\mu .
$$

Furthermore, by (f2) and (f3), there is an $S>0$ such that

$$
|s|<S \quad \Longrightarrow \quad 0 \leqslant f(x, s) \leqslant f_{0}(x)+\delta<\lambda_{*}<\mu \text { for all } x \in \mathbb{R}^{N} \text {. }
$$

On the other hand, by the Sobolev embedding, there is a constant $C>0$ such that $|v|_{\infty} \leqslant C\|v\|_{X}$ for all $v \in X$. Now define $A:=C / S$ and let $(\lambda, v) \in \mathcal{Z}_{n}$ with $\lambda \geqslant \mu$ and $n \geqslant N_{\mu}$. Suppose by contradiction that $\|v\|_{X}>A$. It follows that

$$
\frac{|v(x)|}{\|v\|_{X}^{2}} \leqslant \frac{|v|_{\infty}}{\|v\|_{X}^{2}} \leqslant \frac{C}{\|v\|_{X}}<S \quad \text { for all } x \in \mathbb{R}^{N} .
$$

The equation $F_{n}(\lambda, v)=0$ can be written as

$$
\Delta v(x) v(x)=\left\{\lambda-\left[1-\chi_{n}(x)\right] f_{\infty}(x)-\chi_{n}(x) f\left(x, \frac{v(x)}{\|v\|_{X}^{2}}\right)\right\} v(x)^{2} .
$$

For $|x| \leqslant n, \chi_{n}(x)=1$ and we have

$$
\Delta v(x) v(x)=\left\{\lambda-f\left(x, \frac{v(x)}{\|v\|_{X}^{2}}\right)\right\} v(x)^{2} \geqslant\left\{\mu-f\left(x, \frac{v(x)}{\|v\|_{X}^{2}}\right)\right\} v(x)^{2} \geqslant 0
$$

by (11). For $|x|>n \geqslant N_{\mu}$, we have

$$
\Delta v(x) v(x)=\left\{\lambda-f_{\infty}(x)\right\} v(x)^{2} \geqslant\left\{\mu-f_{\infty}(x)\right\} v(x)^{2} \geqslant 0
$$


by (10). Hence, $\Delta v(x) v(x) \geqslant 0$ for all $x \in \mathbb{R}^{N}$. Using the maximum principle as in the proof of $[17$, Lemma 3.3], it follows that $v(x) \equiv 0$. This contradiction completes the proof.

The following lemma is a first step towards positivity/negativity of solutions.

Lemma 9. For any fixed $n \in \mathbb{N}$, there is an open neighbourhood $U$ of $\left(\lambda_{\infty}, 0\right)$ in $\mathbb{R} \times X$ such that $v^{2}>0$ on $\mathbb{R}^{N}$ for all $(\lambda, v) \in \mathcal{Z}_{n} \cap U$.

Proof. The first part of the proof is similar to that of [3, Lemma 7]. Assuming by contradiction that there is a sequence $\left\{\left(\lambda_{k}, v_{k}\right)\right\} \subset \mathcal{Z}_{n}$ such that $\lambda_{k} \rightarrow \lambda_{\infty}$, $\left\|v_{k}\right\|_{X} \rightarrow 0$ and $v_{k}^{2} \ngtr 0$ on $\mathbb{R}^{N}$, and defining $u_{k}:=v_{k} /\left\|v_{k}\right\|_{X}$, it follows that there exists $\bar{u} \in X$, with either $\bar{u}>0$ or $\bar{u}<0$, such that $u_{k} \rightarrow \bar{u}$ in $X$. We suppose that $\bar{u}>0$, the other case being similar. The analogue of equation (17) of [3] in the present context is

$$
\Delta u_{k}(x)=\left\{\lambda_{k}-f_{\infty}(x)-\chi_{n}(x) g\left(x, v_{k}(x) /\left\|v_{k}\right\|_{X}^{2}\right)\right\} u_{k}(x) .
$$

Since $\lambda_{k} \rightarrow \lambda_{\infty}$, there exists $k_{0}$ such that $\lambda_{k} \geqslant\left(\lambda_{*}+\lambda_{\infty}\right) / 2>\lambda_{*}$ for all $k \geqslant k_{0}$. Furthermore, there exists $R>n$ such that

$$
\lambda_{k}-f_{\infty}(x)-\chi_{n}(x) g\left(x, v_{k}(x) /\left\|v_{k}\right\|_{X}^{2}\right)>0 \text { for }|x|>R \text { and } k \geqslant k_{0} .
$$

Also, since $u_{k} \rightarrow \bar{u}$ uniformly on $\mathbb{R}^{N}$ by the Sobolev embedding, there exist $\delta>0$ and $k_{1} \geqslant k_{0}$ such that $u_{k}(x) \geqslant \delta$ for $|x| \leqslant R$ and $k \geqslant k_{1}$. Since $u_{k}(x) \rightarrow 0$ as $|x| \rightarrow \infty$, the weak maximum principle now implies that $u_{k} \geqslant 0$ on $\Omega:=\left\{x \in \mathbb{R}^{N}:|x|>R\right\}$, for all $k \geqslant k_{1}$. Rewriting (13) as

$$
-\Delta u_{k}+c_{+} u_{k}=c_{-} u_{k}
$$

with $c(x):=\lambda_{k}-f_{\infty}(x)-\chi_{n}(x) g\left(x, v_{k}(x) /\left\|v_{k}\right\|_{X}^{2}\right)$ and $c_{ \pm}:=\max \{ \pm c, 0\}$, it follows by the strong maximum principle [4, Theorem 8.19] that $u_{k}>0$ on $\mathbb{R}^{N}$, for all $k \geqslant k_{1}$. But this contradicts $v_{k}^{2} \ngtr 0$ on $\mathbb{R}^{N}$, and concludes the proof.

The following lemma describes how elements of $\mathcal{Z}_{n}$ can approach the line of trivial solutions. It can be proved in the same way as Lemma 8 of [3].

Lemma 10. Fix $n \in \mathbb{N}$ and let $\left\{\left(\lambda_{k}, v_{k}\right)\right\} \subset \mathcal{Z}_{n}$. Suppose that $v_{k}^{2}>0$ on $\mathbb{R}^{N}$, $\lambda_{k} \rightarrow \lambda \in J$ and $\left\|v_{k}\right\|_{X} \rightarrow 0$. Then $\lambda=\lambda_{\infty}$.

We now describe the main properties of the component $\mathcal{C}_{n}$.

Proposition 11. (i) For any $n \in \mathbb{N}$, if $(\lambda, v) \in \mathcal{C}_{n} \backslash\left\{\left(\lambda_{\infty}, 0\right)\right\}$ then $\lambda \leqslant \lambda_{\infty}$ and $v^{2}>0$ on $\mathbb{R}^{N}$.

(ii) There is a constant $A>0$ such that, for any $\mu \in J$, there exists $N_{\mu} \in \mathbb{N}$ such that

$$
\forall n \geqslant N_{\mu} \quad \inf \mathrm{P}_{\mathcal{C}_{n}}<\mu \quad \text { and } \quad\|v\|_{X} \leqslant A \quad \text { if } \quad(\lambda, v) \in \mathcal{C}_{n} \text { with } \lambda \geqslant \mu .
$$

Proof. The first part of the proof follows that of Proposition 9 in [3], using Lemma 9, Lemma 10 and similar maximum principle arguments as above to show that the set

$$
\mathcal{Q}=\left\{(\lambda, v) \in \mathcal{C}_{n}: v^{2}>0 \text { on } \mathbb{R}^{N}\right\} \cup\left\{\left(\lambda_{\infty}, 0\right)\right\}
$$


is both open and closed in $\mathcal{C}_{n}$, with respect to the topology inherited from $\mathbb{R} \times X$. Since $\mathcal{C}_{n}$ is connected, this implies $\mathcal{C}_{n}=\mathcal{Q}$. Furthermore, using Proposition 3 , it follows by a similar proof to that of [3, Proposition 14(iv)] that solutions of $F_{n}(\lambda, v)=0$ with either $v>0$ or $v<0$ satisfy $\lambda \leqslant \lambda_{\infty}$. This proves (i).

The proof of (ii) is identical to that of Proposition 9(ii) in [3], using Lemma 8 and Lemma 10 above.

We are now in a position to prove global bifurcation for the inverted problem (7). We define $F: J \times X \rightarrow Y$ by

$$
(\lambda, v):=L(\lambda) v+G(v), \quad v \in X,
$$

and we let

$$
\mathcal{Z}:=\left\{(\lambda, v) \in J \times X: v^{2}>0 \text { and } F(\lambda, v)=0\right\},
$$

$\mathcal{C}:=$ connected component of $\mathcal{Z} \cup\left\{\left(\lambda_{\infty}, 0\right)\right\}$ containing the point $\left(\lambda_{\infty}, 0\right)$.

The following estimate is proved in the same way as Lemma 8 , replacing $\chi_{n}$ by 1 .

Lemma 12. Let $A>0$ be the constant given by Lemma 8. For all $(\lambda, v) \in J \times X$ such that $F(\lambda, v)=0$ we have $\|v\|_{X} \leqslant A$.

Theorem 13. Suppose $U \subset \mathbb{R} \times X$ is open, bounded, such that $\left(\lambda_{\infty}, 0\right) \in U$ and

$$
\mu:=\inf \{\lambda:(\lambda, v) \in U\}>\lambda_{*} .
$$

(i) $\mathcal{Z} \cap \partial U \neq \varnothing$.

(ii) $\mathcal{C}$ is bounded in $\mathbb{R} \times X$ with $\inf \mathrm{P} \mathcal{C}=\lambda_{*}$ and $\sup \mathrm{P} \mathcal{C}=\lambda_{\infty}$.

(iii) Let $\left\{\left(\lambda_{k}, v_{k}\right)\right\} \subset \mathcal{C}$. If $\lambda_{k} \rightarrow \lambda \in J$ and $\left\|v_{k}\right\|_{X} \rightarrow 0$, then $\lambda=\lambda_{\infty}$. Conversely, if $\lambda_{k} \rightarrow \lambda_{\infty}$, then $\left\|v_{k}\right\|_{X} \rightarrow 0$.

Proof. Using the preceding results, the proof essentially follows that of Theorem 11 in [3] and we shall only indicate a few details here. First note that, in [3], we were dealing with positive solutions only. However, the limit procedure can be carried out in the same way here, yielding solutions with either $v \geqslant 0$ or $v \leqslant 0$ on $\mathbb{R}^{N}$. It then follows by the strong maximum principle (as used in the proof of Lemma 9) that such solutions actually satisfy either $v>0$ or $v<0$ on $\mathbb{R}^{N}$.

Also, it is required in the proof that solutions of $F(\lambda, v)=0$ with either $v>0$ or $v<0$ satisfy $\lambda<\lambda_{\infty}$. Using the eigenfunction $\varphi_{\infty}>0$ given by Proposition 3, it follows as in the first part of the proof of [3, Proposition 14(iv)] that

$$
\lambda_{\infty} \int_{\mathbb{R}^{N}} \varphi_{\infty} v \mathrm{~d} x=\lambda \int_{\mathbb{R}^{N}} v \varphi_{\infty} \mathrm{d} x-\int_{\mathbb{R}^{N}} h(x, v) v \varphi_{\infty} \mathrm{d} x,
$$

where $h(x, v(x)):=f\left(x, v(x) /\|v\|_{X}^{2}\right)-f_{\infty}(x)$. Assuming that $v>0$ on $\mathbb{R}^{N}$ and remarking that, by (f4), $h \leqslant 0$, we see that $\lambda_{\infty} \geqslant \lambda$. Then, by (f2) and (f3),

$$
\liminf _{|x| \rightarrow \infty} h(x, v(x))=\liminf _{|x| \rightarrow \infty} f_{0}(x)-\lambda_{*} \leqslant-\delta<0 .
$$


Since $h(x, v(x))$ is continuous, it follows that $h(x, v(x))<0$ on a set of positive measure. Hence, $\int_{\mathbb{R}^{N}} h(x, v) v \varphi_{\infty} \mathrm{d} x<0$ and we have $\lambda_{\infty}>\lambda$. The case $v<0$ is similar.

\section{Proof of Theorem 1}

We shall make the hypotheses (f1)-(f6) throughout this section and use the same notations as above.

We prove Theorem 1 using the solution set obtained in Theorem 13 and the inversion $v \rightarrow v /\|v\|_{X}^{2}$. To preserve connectedness under inversion, we first need to get rid of the right end-point of $\mathcal{C}$. This is done by the following lemma, which can be proved similarly to Corollary 5.3 of [17].

Lemma 14. There exists a connected subset $\mathcal{C}_{0}$ of $\mathcal{C} \backslash\left\{\left(\lambda_{\infty}, 0\right)\right\}$ such that $\inf \mathrm{P} \mathcal{C}_{0}=\lambda_{*}$ and $\left(\lambda_{\infty}, 0\right) \in \overline{\mathcal{C}_{0}}$. In particular, $\sup \mathrm{P} \mathcal{C}_{0}=\lambda_{\infty}$ and $0<\|v\|_{X} \leqslant$ $A$ for all $(\lambda, v) \in \mathcal{C}_{0}$.

Remark 2. Since $\mathcal{Z} \subset \mathbb{R} \times C\left(\mathbb{R}^{N}\right)$, it follows that the set $\mathcal{C}_{0}$ obtained in Lemma 14 lies in one of the sets $\mathcal{Z}^{ \pm}:=\{(\lambda, v) \in \mathcal{Z}: \pm v>0\}$.

Using the same anti-symmetrization procedure as in the beginning of the proof of Theorem 2.3 in [17], we obtain two connected subsets $\mathcal{C}_{0}^{ \pm}$of $\mathcal{C} \backslash\left\{\left(\lambda_{\infty}, 0\right)\right\}$, consisting of positive/negative solutions and satisfying the properties of $\mathcal{C}_{0}$ given by Lemma 14 . Then, from the preceding results, we see that setting

$$
\mathcal{S}^{ \pm}:=\left\{\left(\lambda, \frac{v}{\|v\|_{X}^{2}}\right):(\lambda, v) \in \mathcal{C}_{0}^{ \pm}\right\}
$$

defines connected sets of positive/negative solutions of (1). It now follows from Proposition 2 that $\mathcal{S}^{ \pm} \subset \mathbb{R} \times H^{1}\left(\mathbb{R}^{N}\right)$ and that property (iv) of Theorem 1 holds. Furthermore, properties (i) and (ii) follow from Theorem 13(ii). It only remains to prove (iii). We already know from Theorem 13(iii) that $\left\|u_{n}\right\|_{X} \rightarrow \infty \Leftrightarrow \lambda=\lambda_{\infty}$ and, since $-\Delta+1: X \rightarrow Y$ is an isomorphism, it follows from

$$
-\Delta u_{n}+u_{n}=\left[f\left(x, u_{n}^{2}\right)-\lambda_{n}\right] u_{n}+u_{n}
$$

that $\left\|u_{n}\right\|_{Y} \rightarrow \infty \Leftrightarrow\left\|u_{n}\right\|_{X} \rightarrow \infty$. As for $\left|u_{n}\right|_{\infty}$, by the Sobolev embedding, $\left|u_{n}\right|_{\infty} \rightarrow \infty \Rightarrow\left\|u_{n}\right\|_{X} \rightarrow \infty$. For the converse, suppose by contradiction that $\left\|u_{n}\right\|_{X} \rightarrow \infty$ and there is a subsequence $u_{j}:=u_{n_{j}}$ such that $\left|u_{j}\right|_{\infty}$ is bounded. It follows by Lemma 7 that $\left\{u_{j}\right\}$ is bounded in $Y$ and hence, by (15), bounded in $X$. This contradiction concludes the proof of Theorem 1.

\section{References}

[1] Costa, D.G., Tehrani, H.: On a class of asymptotically linear elliptic problems in $\mathbb{R}^{N}$. J. Differ. Equ. 173(2), 470-494 (2001) 
[2] Genoud, F.: Bifurcation and stability of travelling waves in self-focusing planar waveguides. Adv. Nonlinear Stud. 10(2), 357-400 (2010)

[3] Genoud, F.: Bifurcation from infinity for an asymptotically linear problem on the half-line. Nonlinear Anal. 74, 4533-4543 (2011)

[4] Gilbarg, D., Trudinger, N.S.: Elliptic Partial Differential Equations of Second Order, 2nd edn. Springer, Berlin (2001)

[5] Jeanjean, H., Lucia, M., Stuart, C.A.: Branches of solutions to semilinear elliptic equations on $\mathbf{R}^{N}$. Math. Z 230, 79-105 (1999)

[6] Jeanjean, H., Stuart, C.A.: Nonlinear eigenvalue problems having an unbounded branch of symmetric bound states. Adv. Differ. Equ. 4, 639-670 (1999)

[7] Jeanjean, L.: On the existence of bounded Palais-Smale sequences and application to a Landesman-Lazer-type problem set on $\mathbf{R}^{N}$. Proc. Roy. Soc. Edinb. Sect. A 129(4), 787-809 (1999)

[8] Jeanjean, L., Tanaka, K.: A positive solution for an asymptotically linear elliptic problem on $\mathbb{R}^{N}$ autonomous at infinity. ESAIM Control Optim. Calc. Var 7, 597-614 (2002)

[9] Li, G., Zhou, H.-S.: The existence of a positive solution to asymptotically linear scalar field equations. Proc. Roy. Soc. Edinb. Sect. A 130(1), 81-105 (2000)

[10] Liu, Z., Wang, Z.-Q.: Existence of a positive solution of an elliptic equation on $\mathbb{R}^{N}$. Proc. Roy. Soc. Edinb. Sect. A 134(1), 191-200 (2004)

[11] Rabinowitz, P.H.: On bifurcation from infinity. J. Differ. Equ. 14, 462-475 (1973)

[12] Rabier, P.J.: Salter MF A degree theory for compact perturbations of proper $C^{1}$ Fredholm mappings of index 0. Abstr. Appl. Anal. 7, 707-731 (2005)

[13] Stuart, C.A.: An introduction to elliptic equations on $\mathbf{R}^{N}$, in nonlinear functional analysis and applications to differential equations (Trieste, 1997), pp. 237-285. World Science Publishing, River Edge (1998)

[14] Stuart, C.A., Zhou, H.-S.: A variational problem related to self-trapping of an electromagnetic field. Math. Methods Appl. Sci 19(17), 1397-1407 (1996)

[15] Stuart, C.A., Zhou, H.-S.: Applying the mountain pass theorem to an asymptotically linear elliptic equation on $\mathbf{R}^{N}$. Comm. Partial Differ. Equ. 24(9-10), 1731-1758 (1999)

[16] Stuart, C.A., Zhou, H.-S.: Axisymmetric TE-modes in a self-focusing dielectric. SIAM J. Math. Anal. 37(1), 218-237 (2005)

[17] Stuart, C.A., Zhou, H.-S.: Global branch of solutions for nonlinear Schrödinger equations with deepening potential well. Proc. Lond. Math. Soc 92(3), 655-681 (2006)

[18] Toland, J.F.: Asymptotic nonlinearity and nonlinear eigenvalue problem, Quart. J. Math. Oxf. 24, 241-250 (1973) 
[19] Van Heerden, F.A.: Multiple solutions for a Schrödinger type equation with an asymptotically linear term. Nonlinear Anal. 55(6), 739-758 (2003)

[20] Van Heerden, F.A., Wang, Z.-Q.: Schrödinger type equations with asymptotically linear nonlinearities. Differ. Integral Equ. 16(3), 257-280 (2003)

[21] Zhou, H.-S.: Positive solution for a semilinear elliptic equation which is almost linear at infinity. Z. Angew. Math. Phys. 49(6), 896-906 (1998)

[22] Zhou, H.-S., Zhu, H.: Asymptotically linear elliptic problem on $\mathbb{R}^{N}$. Q. J. Math. 59(4), 523-541 (2008)

François Genoud

Maxwell Institute for Mathematical Sciences

Department of Mathematics

Heriot-Watt University

Edinburgh

EH14 4AS

Scotland

e-mail: F.Genoud@hw.ac.uk

Received: 1 July 2011.

Accepted: 17 January 2012. 